\title{
TODO LO QUE QUERIA SABER SOBRE COMO SOLDAR Y NO SE ATREVIA A PREGUNTAR
}

\section{EVERYTHING YOU WANTED TO KNOW ABOUT HOW SOLDIERS AND NOT DARED TO ASK}

\section{AUTORES}

Arturo Pérez París: Alumno de la Escuela Politécnica de la Universidad de Alcalá arturo.perez@mixmail.com

\section{CURRÍCULUM VITAE}

Alumno de la Escuela Politécnica de la Universidad de Alcalá de Henares (España). Ingeniero de Soporte Técnico en Kone Elevadores

\section{RESUMEN}

Hace un tiempo, un año más o menos, me di cuenta de algo que me dejó perplejo: Muchos compañeros míos de carrera no saben hacer soldaduras en componentes o conectores. Por cierto, yo estudio, o por lo menos lo intento siempre y cuando me dejan claro, Ingeniería Técnica de Telecomunicaciones. El evento descrito es normal. Nadie les ha enseñado y como es lógico por "ciencia infusa" no se aprende nada (cuando menos yo aún no lo visto).

\section{PALABRAS CLAVE}

Soldar - Aprender - Preguntar

\section{ABSTRACT}


Some time ago, a year or so, I realized something that puzzled me: Many of my classmates do not know career welds in components or connectors. By the way, I study or at least try as long as I make clear, Telecommunications Engineering. The event described is normal. No one has taught and of course by "infused knowledge" do not learn anything (at least I have not seen).

\section{KEY WORDS}

Welding - Learning - Ask.

Hace un tiempo, un año más o menos, me di cuenta de algo que me dejó perplejo: Muchos compañeros míos de carrera no saben hacer soldaduras en componentes o conectores. Por cierto, yo estudio, o por lo menos lo intento siempre y cuando me dejan claro, Ingeniería Técnica de Telecomunicaciones. El evento descrito es normal. Nadie les ha enseñado y como es lógico por "ciencia infusa" no se aprende nada (cuando menos yo aún no lo visto). Sobre este punto me gustaría hacer una pequeña reflexión antes de continuar con el presente tema:

Algo que muchos deberían tener presente es el hecho de que las personas, salvo raras excepciones, no nacemos sabiendo (esto es lógico, público y notorio). De hecho, esta es una de las cualidades que nos diferencian del resto de los animales y vegetales conocidos. Estos nacen con ciertos "conocimientos" propios de su especie a los que llamamos instintos y una cierta capacidad de aprendizaje basada en éstos. Nosotros, como animales "racionales" (...), nacemos con una capacidad superior de aprender debido a que nuestro bagaje de conocimientos instintivos es prácticamente inexistente, (aunque en ciertos individuos \as no está tan claro), en comparación con 
los "irracionales". Esto en sí nos hace lo que somos: personas, no animales, (le pese a quien le pese).

En fin, retomemos nuestro tema. Cuando vi este problema me dije a mí mismo: Tienes la oportunidad mediante la revista de comunicar lo que se sabes. ¿Por qué no lo hacemos?. Pues bien, espero que el presente escrito ayude a quien lo lea. Ahora, a soldar sólo se aprende practicando, así que recomendaría su ejercicio debido a lo útil que resulta, (sobre todo para la gente de mi gremio), cara al mundo profesional .

La soldadura de metales de fácil fusión, como el plomo y el estaño, es llamada soldadura blanda o estañadura. Normalmente no se emplean el estaño o el plomo solos, sino una aleación de ambos, en las proporciones adecuadas según su fin, (a diferentes tipos de trabajos diferentes tipos de aleaciones). Este tipo de soldadura se emplea cuando la temperatura de las piezas unidas, en su trabajo habitual, no sobrepasa $\operatorname{los} 250^{\circ} \mathrm{C}$. Esta temperatura funde los aludidos metales o su mezcla.

La soldadura blanda se suele emplear en la unión de chapas y piezas de latón, hojalata, cobre o bronce, colectores de inducidos, empalmes, soldadura de tubos y como no en lo que a nosotros más nos interesa: la unión de componentes a circuitos impresos y la de los cables a sus respectivos conectores.

En la soldadura blanda o estañadura se usa generalmente el plomo y el estaño, como se dijo, ahora bien, existen diversas proporciones de estos dos metales según el trabajo a realizar, no es lo mismo soldar componentes electrónicos a un circuito, que soldar dos cañerías de plomo. La aleación a emplear será distinta. En el primer caso, que es el que además nos interesa, la aleación utilizada es del 60\% estaño 40\% plomo, cuyo punto de fusión está entre $\operatorname{los} 180^{\circ} \mathrm{C}$ y $\operatorname{los} 230^{\circ} \mathrm{C}$. Para el segundo caso la proporción es diferente e incluso nos lo podemos encontrar con plata que aumenta la dureza de la soldadura y el punto de fusión, mas esto no es lo que necesitamos. La presentación de las aleaciones es diversa, según las necesidades a las que esté destinada, a saber:

- En hilos bobinados en carretes, que será el que habitualmente utilizaremos en las soldaduras sobre circuito impreso, regletas y conectores. 
- Varillas sueltas para soldar con lámpara de butano.

- Bolas para máquinas automáticas de soldar y cubetas de soldadura por ola

- Discos y arandelas para aplicaciones especiales.

\section{m}
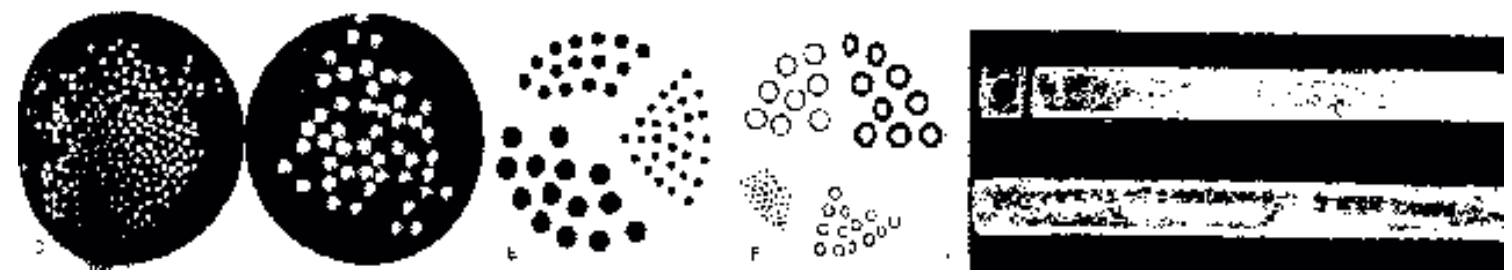

Formas de presentación de las aleaciones de estaño: A, en carretes; B, en varillas; C, en lingotes; D, en bolas; E, en discos; F, en arandelas.

La adherencia del estaño (que será como coloquialmente denominaremos a la aleación) sobre el material a soldar no es posible sin el empleo de un material fundente-desoxidante. Para nuestras aplicaciones será una resina o pasta que no sean de tipo ácido, ya que si así fuere se corroería el cobre de las placas o los pines de los componentes. Normalmente los hilos de aleación de estaño para soldar, que realidad son tubitos huecos, llevan en su interior una pequeña cantidad de esta pasta desoxidante a la que se denomina "alma".

Del soldador, además de tener la capacidad de producirnos cuantiosas quemaduras al calentarse (sobre todo en aquellos individuos/as poco hábiles), las características que lo determinan son la potencia y el tipo de punta para soldar; además de las propiedades mecánicas comunes de toda herramienta. Existe una amplia gama de modelos de este útil, desde los más sencillos y baratos de lapicero hasta los más sofisticados y caros capaces de depositar automáticamente el estaño al soldar. Los prototipos que habitualmente nos encontraremos son: 
- Soldador estándar. Se construye para potencias comprendidas entre 35 y 200 watios para tensiones de 12, 24, 125 y 220 voltios (este es el más común de todos).

\section{Soldador eléctrico}

- Soldador miniatura, también llamado de lápiz. Se construye para potencias comprendidas entre 40 y 60 watios para tensiones de $12,24,125$ y 220 voltios. Estos son los más apropiados para los trabajos de electrónica, siendo la mayoría de puntas intercambiables.
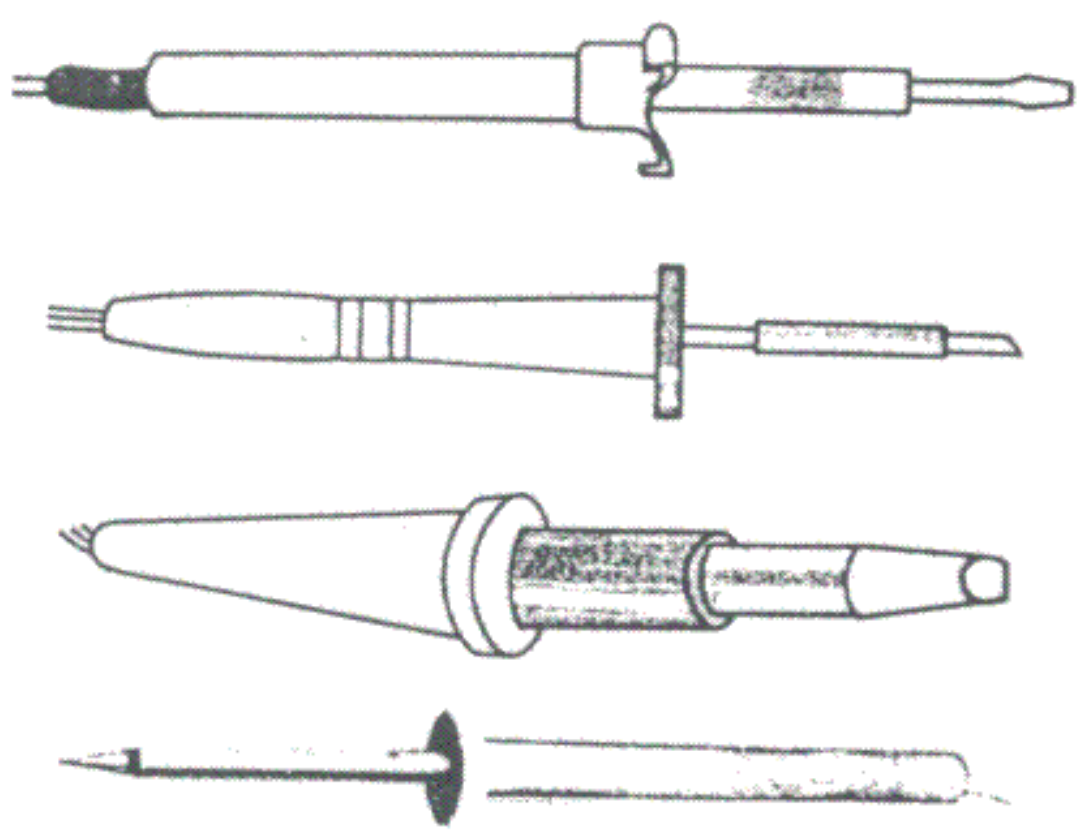

Soldadores Miniatura

- Soldadores de temperatura controlada. Son aquellos que disponen de un mecanismo que estabiliza la temperatura de la punta, permitiendo que ésta permanezca a una temperatura constante, aún en condiciones muy duras de trabajo. . Se construye para potencias comprendidas entre 20 y 100 watios para tensiones de 24,125 y 220 voltios.

Corte de un soldador de temperatura constante 
Aspecto externo de un soldador de temperatura constante

- Soldadores de pistola. Son de calentamiento instantáneo. Para ello, se hace circular una corriente de alto valor por la punta, con lo que ésta en muy poco tiempo adquiere la temperatura de régimen permanente. Su uso está muy generalizado. No resulta adecuado en trabajos de montaje ni, en general, en aquellos trabajos que requieren una frecuencia de soldadura media o alta. Se fabrican en una amplia gama de potencias y para tensiones de 125 o $220 \mathrm{~V}$.

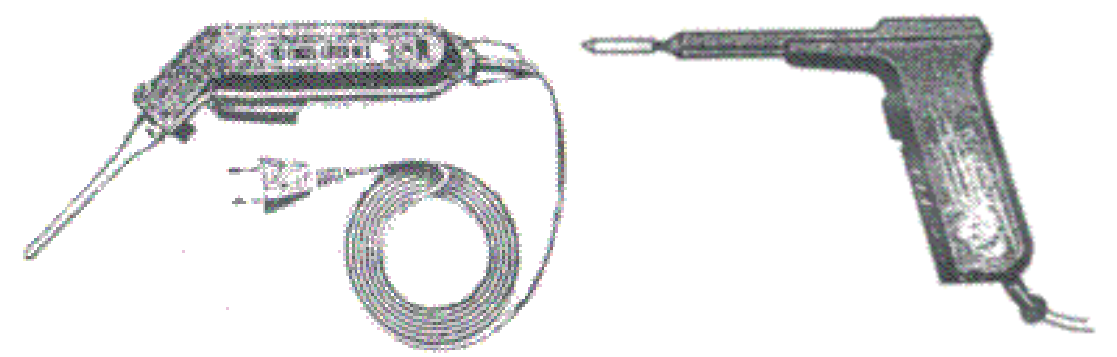

Soldadores de pistola

- Soldadores de martillo. De gran potencia (entre 100 y $500 w$ ) resultan adecuados para aquellos trabajos que requieran un gran aporte de calorías. (por ejemplo grandes placas, conductores gruesos, etc.).

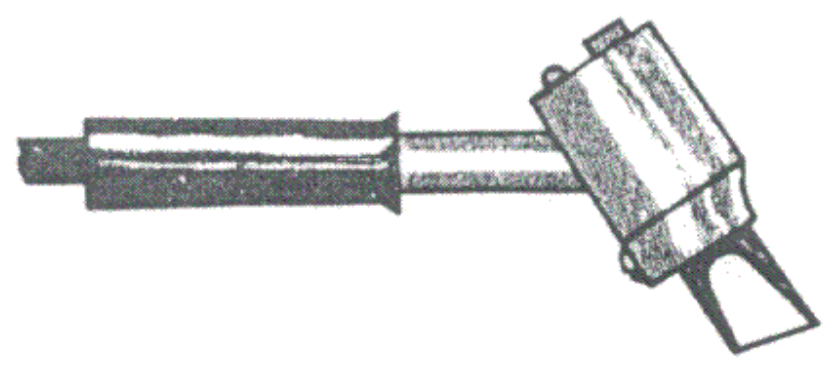

Soldador de martillo 
- Soldadores automáticos. Al depositar automáticamente el estaño garantizan la uniformidad de las soldaduras. Resultan adecuados en grandes cadenas de producción y además permiten al operario tener una mano libre durante la realización de la soldadura.

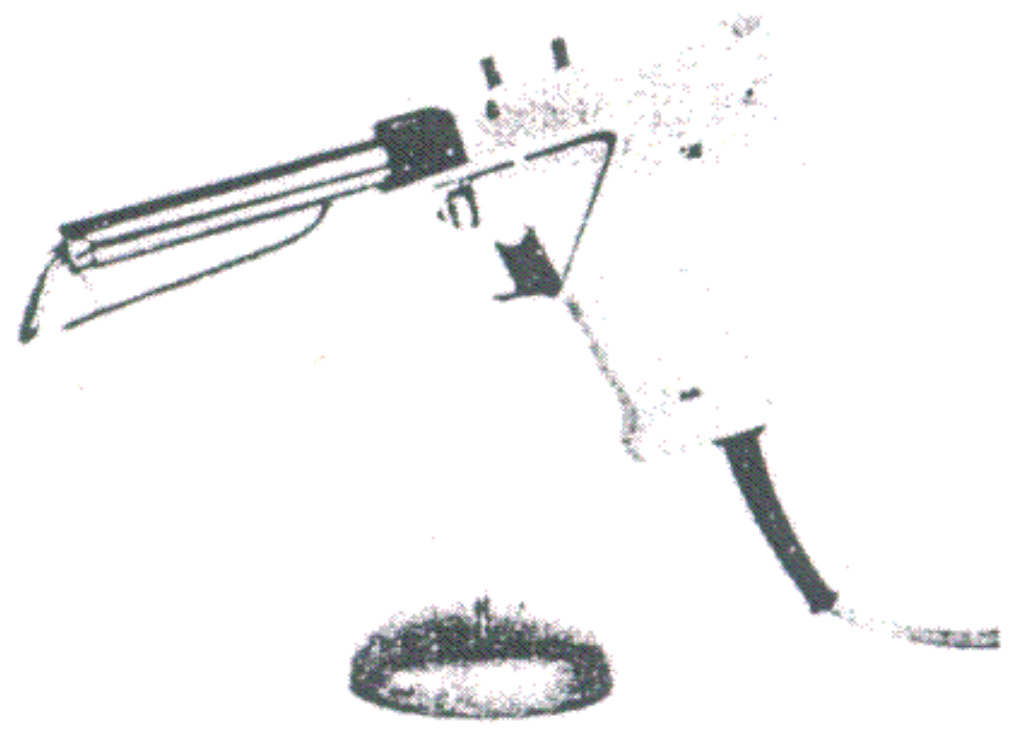

Soldador automático

La siguiente tabla nos muestra el soldador a usar según el trabajo a realizar:

\begin{tabular}{|c|c|c|}
\hline $\begin{array}{c}\text { Potencia } \\
\text { (Watios) }\end{array}$ & $\begin{array}{c}\text { Diámetro de } \\
\text { la punta(mm) }\end{array}$ & Utilización \\
\hline $15-20$ & 3 & $\begin{array}{c}\text { Soldaduras de circuitos integrados, circuitos } \\
\text { impresos, transistores y elementos que } \\
\text { soportan poca cantidad de calor. }\end{array}$ \\
\hline $30-40$ & 4 & $\begin{array}{c}\text { Soldaduras en circuitos eléctricos, radio y } \\
\text { TV con conductores de sección inferior a 0.5 } \\
\mathrm{mm}^{2} \text {. }\end{array}$ \\
\hline 60 & 5.5 & $\begin{array}{c}\text { Soldadura en circuitos eléctricos, radio y TV } \\
\text { con conductores de sección superior a 0.5 } \\
\text { mm² y bases metálicas de espesor inferior a } \\
\text { los } 0.2 \text { mm }^{2} .\end{array}$ \\
\hline 80 & 8 & $\begin{array}{r}\text { Soldadura y conexionado de conductores } \\
\text { entre sí y sobre bases metálicas con } \\
\text { espesor de hasta } 0.3 \mathrm{~mm}^{2} \text {. }\end{array}$ \\
\hline
\end{tabular}




\begin{tabular}{|c|c|c|}
\hline $100-120$ & 10 & $\begin{array}{c}\text { Soldaduras de conductores entre sí con } \\
\text { componentes y sobre chasis. }\end{array}$ \\
\hline 150 & 14 & $\begin{array}{c}\text { Soldaduras sobre chasis o láminas metálicas } \\
\text { de hasta } 0.8 m \text { m de espesor, empalmes y } \\
\text { bañado de conductores, conexión en } \\
\text { pequeños colectores de motores, etc. }\end{array}$ \\
\hline 200 & 16 & $\begin{array}{c}\text { Soldadura de hilos con secciones no } \\
\text { superiores a los } 6 \mathrm{~mm}^{2}, \text { piezas metálicas y } \\
\text { chapas, bañado de piezas, soldaduras de } \\
\text { terminales y colectores, etc. (potencia). }\end{array}$ \\
\hline
\end{tabular}

Gran número de soldadores posee puntas intercambiables, multiplicando así la disponibilidad de estos instrumentos. Las puntas son de cobre, para permitir una buena conducción del calor. Este metal tiene el inconveniente de que es atacado y disuelto por el estaño líquido y el fundente. Pronto se notan orificios diminutos y pequeñas irregularidades en la aleta de soldar, lo que da lugar a soldaduras pobres, por ello las mejores llevan un recubrimiento de aleaciones de hierro, aluminio, cromo y níquel. Al bañar la punta se estará pendiente de la temperatura, comprobando cuando funde el estaño, momento en el que se procederá a recubrirla. Esta operación se realiza fundiendo en la punta, por contacto, un poco de resina. Posteriormente se aplica estaño que se frota con un trapo limpio (hay que procurar que éste no sea de un material plástico como el nylon, ya que al ser plástico se derretiría en la punta guarreándola). Es preciso notar que, estando el soldador muy caliente, se adhiere mal el estaño, por lo cual, como antes quedó indicado, la operación del baño se debe realizar en el instante en que la temperatura alcanzada empieza a fundir el estaño.

Muy importante para realizar buenas soldaduras es que la punta esté lisa y perfectamente limpia, exenta de residuos de estaño o resina quemada. Por esto, cuando se suelde se tendrá siempre a mano un paño limpio o 
esponjas destinadas para tal fin (p.e. las que suelen traer los soportes para los soldadores).
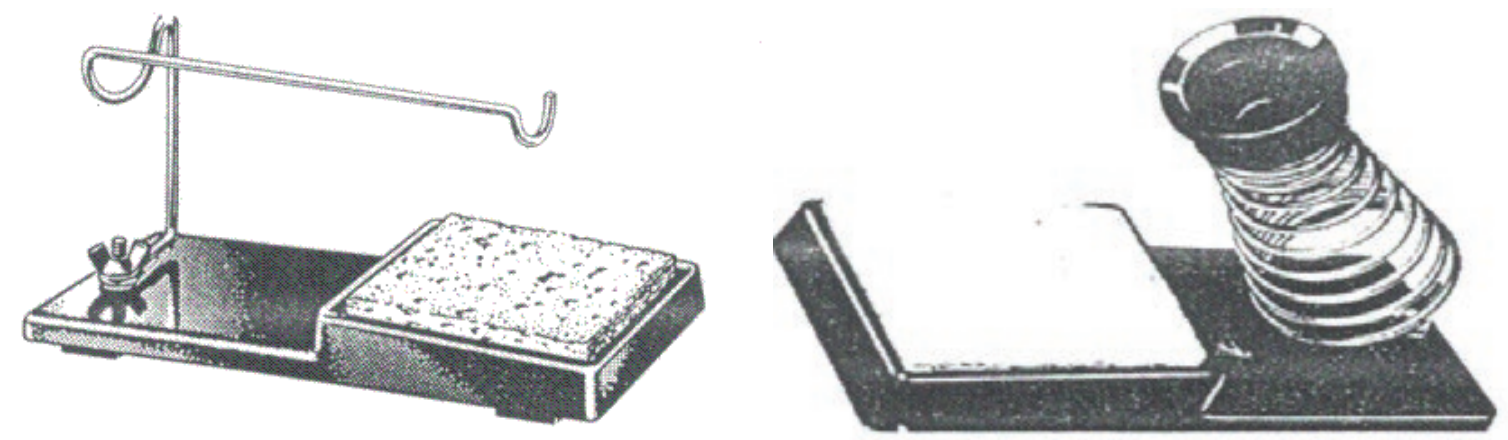

Soportes de soldador y esponjas para limpiar las puntas

Una vez elegidos el soldador y el estaño apropiados, para la soldadura que se realizará, interesa ver cómo deben realizarse las soldaduras.

Estando escrupulosamente limpios los elementos por soldar, se aplica el soldador al punto en el que se va a efectuar la soldadura para dar calor a la zona. Sin este calor previo, se corre el riesgo de que la soldadura quede mal o lo que en el argot de la profesión se denomina soldadura fría. Una vez caliente la zona que se desea soldar, se aplica el estaño. Si las operaciones anteriores se han realizado correctamente, el estaño se extenderá por la zona que ha de soldarse. Cuando suceda esto, retírese rápidamente y a un tiempo estaño y soldador. La soldadura aparecerá limpia, brillante y redondeada al primer intento (...).

A pesar de que ésta es la forma general de efectuar las soldaduras, son necesarias ciertas condiciones para cuando se efectúa la soldadura de los siguientes elementos:

- Soldaduras de hilos y cables. Normalmente los utilizados en electrónica ya están preparados para esta circunstancia, pues han sido sometidos a un estañado previo. Igual tratamiento han recibido los terminales de los componentes electrónicos. Cuando los hilos sean de cobre sin recubrimiento, se tendrá la precaución de raspar 
los trozos que se van a soldar (con la hoja de una tijera por ejemplo), para eliminar residuos de óxido que dificultarían la soldadura. Igual precaución hay que tomar cuando se van a soldar hilos de bobinas, pues éstos van recubiertos de esmalte aislante. En electrónica muchas veces hay necesidad de soldar hilos o terminales al chasis; para ello se raspará la zona del chasis donde se va a efectuar la soldadura y después se efectuará ésta con un soldador de por lo menos $75 \mathrm{~W}$.

- Para soldaduras de transistores, diodos, etc. se elegirá un soldador que no sobre pase los $50 \mathrm{~W}$ de potencia. Para favorecer la evacuación de calor del componente, se tomará la patilla que se ha de soldar con unas pinzas o unos alicates realizando la soldadura en el menor tiempo posible (esto además evitará que nos quememos los dedos).

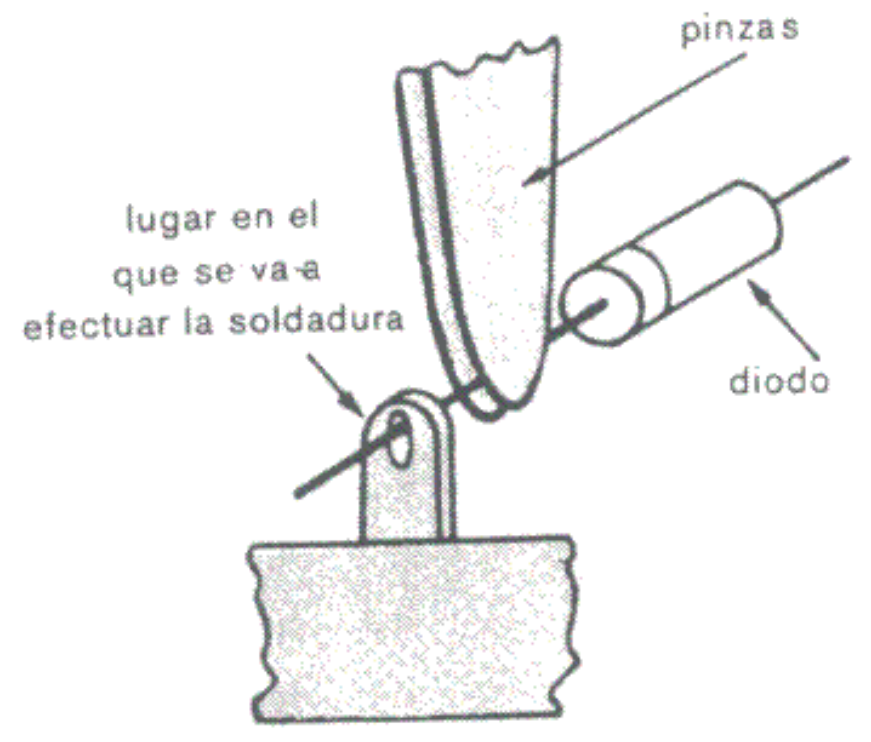

Soldadura de un diodo

- La soldadura de circuitos impresos es la situación más delicada, pues no sólo se corre el riesgo de destruir el componente por un exceso de calor, 
sino que también puede destruirse el mismo circuito impreso. Por todo ello se pondrá especial cuidado en las normas dichas anteriormente, el soldador tampoco superará los $50 \mathrm{~W}$ de potencia. Los circuitos impresos deben cogerse por los borde sin tocar las pistas de cobre, pues ello podría ocasionar su oxidación. Si las pistas se encuentran oxidadas cuando se van a soldar, se limpiarán con productos a propósito que se venden en el comercio o, en caso de necesidad con goma de borrar tinta. Prescíndase del uso del alcohol, ya que éste disuelve la laca protectora del circuito.

Por último, se añadirá que son muchos los accesorios que facilitan la operación de soldar o de desoldar: alicates, pinzas, tijeras, etc. Por ello, es recomendable acercarse a los comercios especializados con el fin de conocerlos. No obstante, cabe destacar algunos que se usan más específicamente en estos menesteres:

- Los portasoldadores y esponjas de limpieza, que ayudan a evitar quemaduras cuando hay que soldar un sitio fijo y que éste se encuentre siempre limpio.

- Las pinzas especiales de extracción, que se fabrican de diversos tipos y resultan muy útiles cuando se trata de extraer circuitos integrados e incluso transistores.

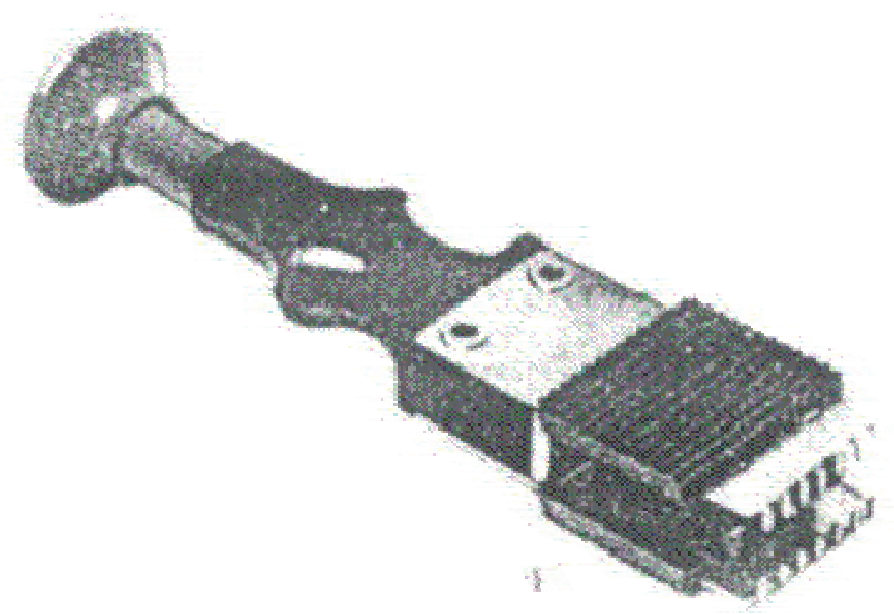

Pinzas para extracción de circuitos integrados 
Con esto doy por finalizado el presente artículo; mas antes quisiera agradecer a $D^{a}$. Matilde París del Pozo su colaboración en este escrito, sin la cual éste no hubiese sido posible. Espero que haya resultado del gusto del lector; si así no hubiera sido, desde aquí hago propósito de enmienda para que el próximo salga mejor (eso si no me echan antes por "rollista" ). Si por el contrario le gustó (al más puro estilo Shakespeariano), quedemos como amigos y volvamos a encontrarnos donde la diosa fortuna más le complazca. Quizás esta manera de despedirse termine convirtiéndose en todo un clásico. El tiempo lo dirá. 